\title{
Avaliação dos fatores associados ao uso de área verde em uma cidade brasileira: um estudo transversal
}

\author{
Assessment of the associated factors to the green area's attendance in \\ a brazilian city: a cross-sectional study
}

Valeska Ribeiro Marques *

Luiz Felipe Silva**

\begin{abstract}
Resumo
As áreas verdes (AV) contribuem para a melhoria da qualidade de vida, podem reduzir temperaturas extremas, atenuar o ruído, reter poeira, aumentar a permeabilidade da área urbana, oferecendo um espaço agradável para a atividade física e de interação social, entre muitos outros benefícios. É um espaço que se comporta como um promotor da saúde. O objetivo deste estudo foi avaliar quais variáveis estão associadas com o uso de AV em Mogi Mirim, SP. Foi realizado um estudo de base populacional, transversal, para verificar os valores das Razões de Chance (RC) de variáveis significativas associadas com a frequência de AV. Os dados foram coletados, por amostragem aleatória de residências, por meio de questionário estruturado a 262 adultos, no período entre fevereiro e agosto de 2012. A técnica utilizada foi a regressão logística para descrever a associação entre a variável dependente, o uso de AV pelo menos uma vez por semana e o conjunto de variáveis explanatórias. Dos entrevistados, $47 \%$ da amostra frequentam AV, pelo menos uma vez por semana. As variáveis que apresentaram associação positiva significativa com o uso de AV foram: ter um cão (RC = 1,88; IC 95\%: 1,13-3,15) e, próprio jardim em casa (RC = 1,83; IC 95\%: 1,08-3,11 A variável que mostrou associação negativa significativa foi utilizar o ônibus como principal meio de transporte (RC=0,51; IC 95\%: 0,29 - 0,89. Esta pesquisa explorou e apresentou uma metodologia de pesquisa para avaliação das características socioeconômicas da população e possíveis associações com uso de AV. Assim, trata-se de um recurso relevante, pouco encontrado na literatura nacional, que deve ser reproduzido com o fim de estimular esta prática essencial de promoção à saúde.
\end{abstract}

Palavras-chave: Áreas verdes urbanas. Estudo de base populacional, Promoção da saúde

\begin{abstract}
Green areas (GA) contribute to improving the quality of life, can reduce temperature extremes, lessen the perception of noise, retain dust, and increase the permeability of the urban area, offering a nice space for physical activity and social interaction between many other benefits. It is a space that behaves as a promoter of health. The aim of this study was to evaluate which variables are associated with the GA attendance in Mogi Mirim, SP. It was conducted a crosssectional study to verify the values of the Odds Ratios of significant variables associated with the GA attendance. Data were collected by random sampling of households, through structured questionnaire to 262 adults, between February and August 2012. The technique applied was logistic regression to describe the association between the dependent variable, AV attendance at least once a week and the set of explanatory variables. Of the respondents, $47 \%$ of the sample attends GA, at least once a week. Variables that showed significant positive association with the GA use were: have $\operatorname{a~dog}(\mathrm{OR}=1.88 ; 95 \% \mathrm{Cl}: 1.13-3.15)$ and, own garden at home $(\mathrm{OR}=1.83 ; 95 \% \mathrm{Cl}: 1.08-3.11$. The variable that showed a significant negative association was use the bus as a primary means of transportation $(\mathrm{OR}=0.51 ; 95 \% \mathrm{Cl}$ : 0.29 - 0.89. This research explored and presented a research methodology for assessing the socioeconomic characteristics of the population and possible associations with use of GA. Thus, it is an important resource, little found in the national literature, which must be replicated in order to stimulate this essential practice of health promotion..
\end{abstract}

Keywords: Urban green areas. Population based study. Health promotion

DOI: 10.15343/0104-7809.20164003372381

\footnotetext{
*Universidade Federal de Itajubá, Itajubá - MG, Brasil. E-mail: valeska.dri@gmail.com **Universidade Federal de Itajubá, Itajubá - MG, Brasil. E-mail: Ifelipe.unifei@gmail.com Os autores declaram não haver conflitos de interesse.
} 


\section{INTRODUÇÃO}

A presença de áreas verdes (AV) está associada com a contribuição para a qualidade de vida da população em espaços urbanos, onde $84,35 \%$ da população vivem ${ }^{1}$. Uma série de benefícios à saúde ao bem-estar pode ser alcançada pela frequência destes espaços.

Não há consenso na definição de AV, induzindo ao uso de terminologias errôneas como sinônimas: espaços livres, área de lazer, espaços verdes, verde urbano e arborização². A quantificação e qualificação da área são importantes, entendendo que AV é um espaço livre, de solo permeável e vegetação arbórea e arbustiva, desde que exerça minimamente função ecológica, estética e de lazer, podendo ser de acesso público ou não ${ }^{3}$. AV tem diferentes funções dependendo do tipo de uso a que se propõe, onde a função social está relacionada ao lazer; às funções estética e ecológica; ao embelezamento da cidade; à promoção de melhorias no clima urbano (ar, água e solo); à função educativa, associada à educação ambiental; à função psicológica, visando o alívio das tensões diárias; ao lazer, recreação e à contemplação ${ }^{4}$.

De modo objetivo e sucinto, AV pode ser delineada como local onde há o predomínio de vegetação arbórea, contemplando assim, praças, jardins públicos e parques urbanos ${ }^{5}$.

Há diversos estudos sobre o tema, pelos quais os desfechos positivos do uso de áreas verdes têm sido abordados. Tais estudos contribuem para a inclusão, no planejamento territorial, da melhoria e da elevação de áreas verdes urbanas ${ }^{6}$. Observa-se que no contexto em havido uma expressiva preocupação em ampliar o uso destes espaços.

O investimento em áreas verdes representa uma política clara de promoção da saúde. Estes espaços podem contribuir para abrandar o nível de estresse, estimulando o convívio social e a prática de esportes ${ }^{7}$.

Há benefícios ambientais oferecidos pelas áreas verdes, que exercem influência favorável sobre a saúde da população urbana, como a possibilidade de atenuação da percepção do nível de ruídos, retenção de poeira, aumento da área permeável urbana, diminuição da temperatura, ambientação de pássaros e beleza inserida na paisagem ${ }^{8,9}$.

Diversos fatores estão associados ao uso das áreas verdes, como: idade, escolaridade, nível social, saúde, distância da residência, ter filhos pequenos, entre outros. Os indivíduos percebem as áreas verdes de modos distintos e a compreensão do que é oferecido está associada também a aspectos culturais ${ }^{8,9}$.

O objetivo deste trabalho foi o de verificar as variáveis associadas à frequência de áreas verdes na cidade de Mogi-Mirim, SP, Brasil.

\section{MATERIAIS E MÉTODOS}

O estudo, observacional de característica transversal, de base populacional, foi conduzido no município de Mogi Mirim, com 86.505 habitantes, localizado na região nordeste do estado de São Paulo, latitude $22,43^{\circ}$ sul e longitude $46,95^{\circ}$ oeste(Figura XX). O Índice de Desenvolvimento Humano Municipal (IDHM), em 2010, é de 0,784, considerado elevado1. A altitude média é de 590 metros.

O clima é controlado por sistemas tropicais e equatoriais, que se caracterizam por alternarem períodos secos de abril a setembro, e úmidos de outubro a março, com temperatura média anual de $20,7^{\circ} \mathrm{C} 10$. 


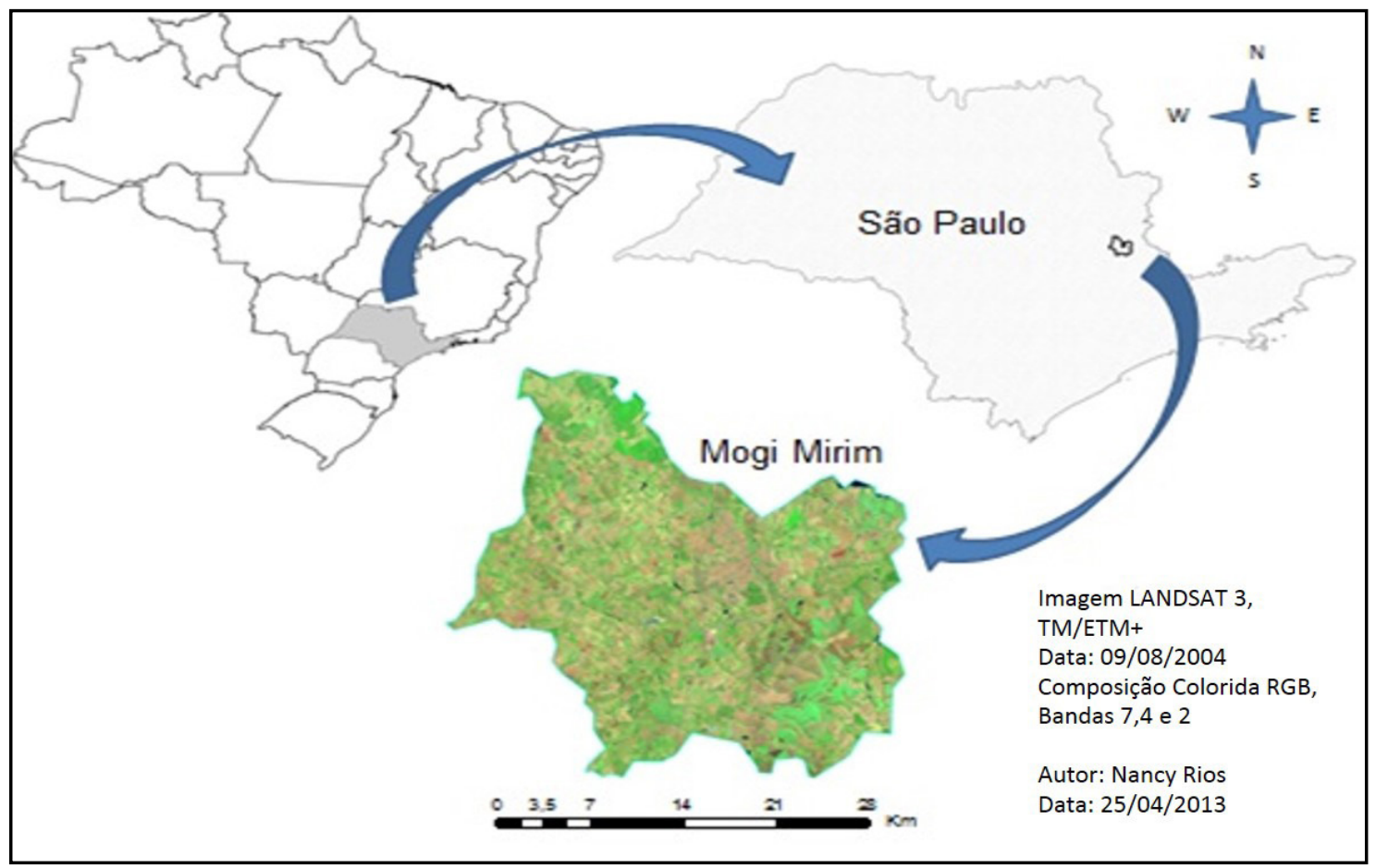

Figura 1: Localização geográfica do município de Mogi Mirim

Área verde foi definida como um local onde há o predomínio de vegetação arbórea, englobando as praças, os jardins públicos e os parques urbanos ${ }^{5}$.

Foram aplicados questionários estruturados em uma amostra da população, pelos quais foram obtidos dados sociodemográficos e comportamentais, buscando identificar as variáveis que explicam a frequência ou não de áreas verdes. Um pré-teste da aplicação do questionário foi conduzido a uma amostra de 15 indivíduos, com o objetivo de corrigir eventuais erros de formulação, tais como: questões complexas e inconsistentes, inacessibilidade ou ambiguidade na linguagem usada, redundância ou embaraço nas questões.

$\mathrm{Na}$ aplicação do questionário, uma questão aberta foi direcionada para que o sujeito entrevistado identificasse áreas verdes frequentadas em Mogi Mirim. Para o calculo da distância da área verde mais frequentada, foi empregado o programa ArcGIS 10.0 partindo das coordenadas da AV de referência à residência do entrevistado. Os valores da distância foram separados em intervalos de 500 metros.

Um grupo de alunos do curso Técnico em Meio Ambiente da Escola Técnica Estadual (ETEC) Pedro Ferreira Alves foram capacitados para contribuir na aplicação dos questionários, com o acompanhamento dos respectivos professores. A classificação do Índice de Massa Corporal (IMC) foi fundamentada nos critérios estabelecidos pelo Ministério da Saúde ${ }^{11}$.

A amostra, de base populacional, foi estabelecida considerando populações finitas, conforme Equação 1:

$$
n=\frac{\delta^{2} \cdot p \cdot q \cdot N}{e^{2}(N-1)+\delta^{2} \cdot p \cdot q}
$$

Onde:

$\mathrm{n}=$ tamanho da amostra: número de entrevistas a serem $\delta 2$ realizadas; 2 = estimativa do nível de confiança determinado, expresso em números de desvio-padrão; $p=$ probabilidade de ocorrência do fenômeno, frequentar área verde; $q=1-p$, número de fracassos; $N=$ dimensão da população, número de habitantes; e = erro máximo permitido ${ }^{12}$. 
Foram adotados dois erros-padrão, valor de $\mathrm{p}$ $=0,5$ e erro máximo permitido de $6 \%$, resultando em uma amostra de 277 domicílios $^{12}$. Foram definidos 318 domicílios, considerando uma margem de segurança de $10 \%$ e adicionando $5 \%$ para eventuais recusas ou perdas. A escolha das residências foi realizada de modo aleatório e proporcional entre os mapas dos setores censitários do município. Os domicílios permanentemente desocupados e os domicílios encontrados fechados foram substituídos pelos imediatamente subsequentes, obedecendo aos mapas setorizados do IBGE'.

As entrevistas foram realizadas entre fevereiro e agosto de 2012. Em cada domicílio selecionado foi entrevistado um morador adulto (com 18 anos de idade ou mais), após explicação sobre o objetivo da pesquisa os entrevistados assinaram voluntariamente $\mathrm{O}$ Termo de Consentimento Livre e Esclarecido, para realização da aplicação do questionário padronizado, que tratou sobre a percepção sobre saúde, hábitos, características pessoais e outras variáveis possivelmente associadas à frequência de uso de áreas verdes. Foram excluídos os indivíduos que residiam há menos de dois anos no município, para evitar influência da pouca adaptação e não conhecimento das opções de áreas verdes do município.

Para descrever a associação entre variável dependente (uso de área verde ou não) e o conjunto de variáveis explanatórias ou preditivas (idade, gênero, escolaridade, meio de transporte, ter filhos, auto avaliação da saúde, uso de medicamento para hipertensão e colesterol, tabagismo, ter cão, ter área verde em casa e índice de massa corporal) foi empregada a técnica de regressão logística multivariada não condicional. As variáveis, identificadas como significativas na análise univariada com valor de $p<0,20$, foram incluídas no modelo utilizando o método progressivo passo a passo (stepwise forward) que incluiu as variáveis independentes uma de cada vez. A permanência das variáveis, para a definição do modelo mais bem ajustado, obedeceu ao critério de p menor ou igual 0,05 fundamentado no teste da máxima verossimilhança ${ }^{13}$.

O logito, ou o log do odds da variável dependente, do modelo de regressão logística é dado pela Equação 2:

Onde:

$$
g(x)=\beta_{0}+\sum_{i=1}^{n} \beta_{i} x_{i}
$$

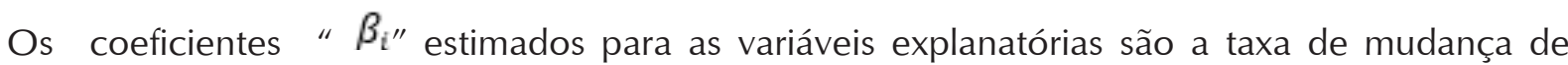
uma função da variável dependente por unidade de mudança na variável explanatória. A partir do conjunto de dados, cada coeficiente é estimado pelo método da verossimilhança e oferece uma estimativa do logaritmo natural (In) da Razão de Chances (RC), com ajuste para as demais variáveis compreendidas no modelo, permitindo assim, estimar diretamente o valor de RC pelo valor do coeficiente $\beta_{i^{\prime}}$, conforme Equação 3:

$$
R C=e^{\beta_{i} X_{i}}
$$

A probabilidade de ocorrência da variável dependente ( $Y=1 /$ Frequência de área verde) foi calculada pela Equação 4:

$$
\operatorname{Prob}(\gamma=1)=\frac{1}{1+e^{-g(x)}}
$$

As análises foram realizadas com auxilio do Epi-info versão 3.5.2.

A pesquisa foi aprovada pelo Comitê de Ética em Pesquisa, no mês de novembro de 2011, sob o parecer de número 744/2011, conforme Resolução 196/96 do Conselho Nacional de Saúde. 


\section{RESULTADOS}

Foram realizadas 318 entrevistas das quais $262(82 \%)$ foram consideradas válidas para a pesquisa, e 56 (18\%) foram descartadas. Foram desconsiderados 45 (14\%) questionários por falta de assinatura do entrevistado, nove (3\%) por residirem há menos de dois anos em Mogi Mirim e dois $(0,5 \%)$ por conterem informações incompletas.

A Tabela 1 apresenta as características dos entrevistados, segundo as variáveis explanatórias contidas no questionário. Não foram encontradas diferenças significativas na idade e na escolaridade entre homens e mulheres.

Tabela 1 - Características da amostra entrevistada, entre fevereiro e agosto de 2012 na cidade de Mogi-Mirim, SP, segundo as variáveis explanatórias.

\begin{tabular}{lc}
\hline \multicolumn{1}{c}{ Variáveis } & Valores \\
\hline Idade (Média \pm DP) & \\
\hline Anos & $46,3 \pm 15,8$ \\
\hline Gênero (\%) & $139(46,9)$ \\
\hline Feminino & $123(53,1)$ \\
Masculino & $90(34,4)$ \\
\hline Escolaridade (\%) & $121(46,2)$ \\
\hline Fundamental & $51(19,5)$ \\
\hline Médio & $6(2,3)$ \\
Superior & $104(39,7)$ \\
\hline íMC (\%) & $97(37,0)$ \\
\hline Abaixo do peso & $55(21,0)$ \\
\hline Peso normal & $239(91,2)$ \\
Acima do peso & $23,8)$ \\
\hline Obeso & \\
\hline Auto avaliação da saúde (\%) & \\
\hline Boa ou muito boa & \\
\hline Ruim & \\
\hline
\end{tabular}

continua... ...continuação - Tabela 1

\begin{tabular}{lc}
\hline Ter filho de até 10 anos (\%) & $66(25,2)$ \\
\hline Posse de cão (\%) & $153(58,4)$ \\
\hline $\begin{array}{l}\text { Meio de Locomoção } \\
\text { no cotidiano (\%) }\end{array}$ & $83(31,7)$ \\
\hline A pé & $116(44,3)$ \\
Automóvel & $78(29,8)$ \\
Ônibus & $19(7,3)$ \\
Moto & $32(12,2)$ \\
\hline Bicicleta & $50(19,1)$ \\
\hline Fumante (\%) & $58(22,1)$ \\
\hline $\begin{array}{l}\text { Usa medicamento } \\
\text { hipertensão (\%) }\end{array}$ & $26(9,9)$ \\
\hline $\begin{array}{l}\text { Usa medicamento } \\
\text { colesterol elevado (\%) }\end{array}$ & $123(46,9)$ \\
\hline Ter "área verde" em casa (\%) & $90(34,4)$ \\
\hline Jardim & $29(11,1)$ \\
Horta & $24(9,2)$ \\
Gramado & $24(9,2)$ \\
\hline $\begin{array}{l}\text { Pomar } \\
\text { Frequência de uso de área } \\
\text { verde (\%) }\end{array}$ & \\
\hline Mínimo uma vez por semana & \\
\hline
\end{tabular}

Dos que frequentam áreas verdes no mínimo uma vez por semana, das 237 respostas, 79 $(33,3 \%)$ o fazem por convívio social; $77(32,5 \%)$ para exercer alguma atividade física; 57 (24,1\%) com o fim de lazer para a criança e $24(10,1)$ para passear com o cão.

Dos 123 entrevistados, que frequentam AV pelo menos uma vez por semana, 60 (48,8\%) referiram o parque público municipal, conhecido como Complexo Lavapés, como local de destino. Das demais respostas 54,3\% se distribuíram entre praças distintas e outros locais. Verificou-se que dos frequentadores do Complexo Lavapés, 90\% deles residiam a até 3 km de distância (Figura 2).

Todas as variáveis explanatórias foram categorizadas, com exceção de idade. Os resultados na análise univariada estão expostos na Tabela 1, em ordem decrescente de significância. 


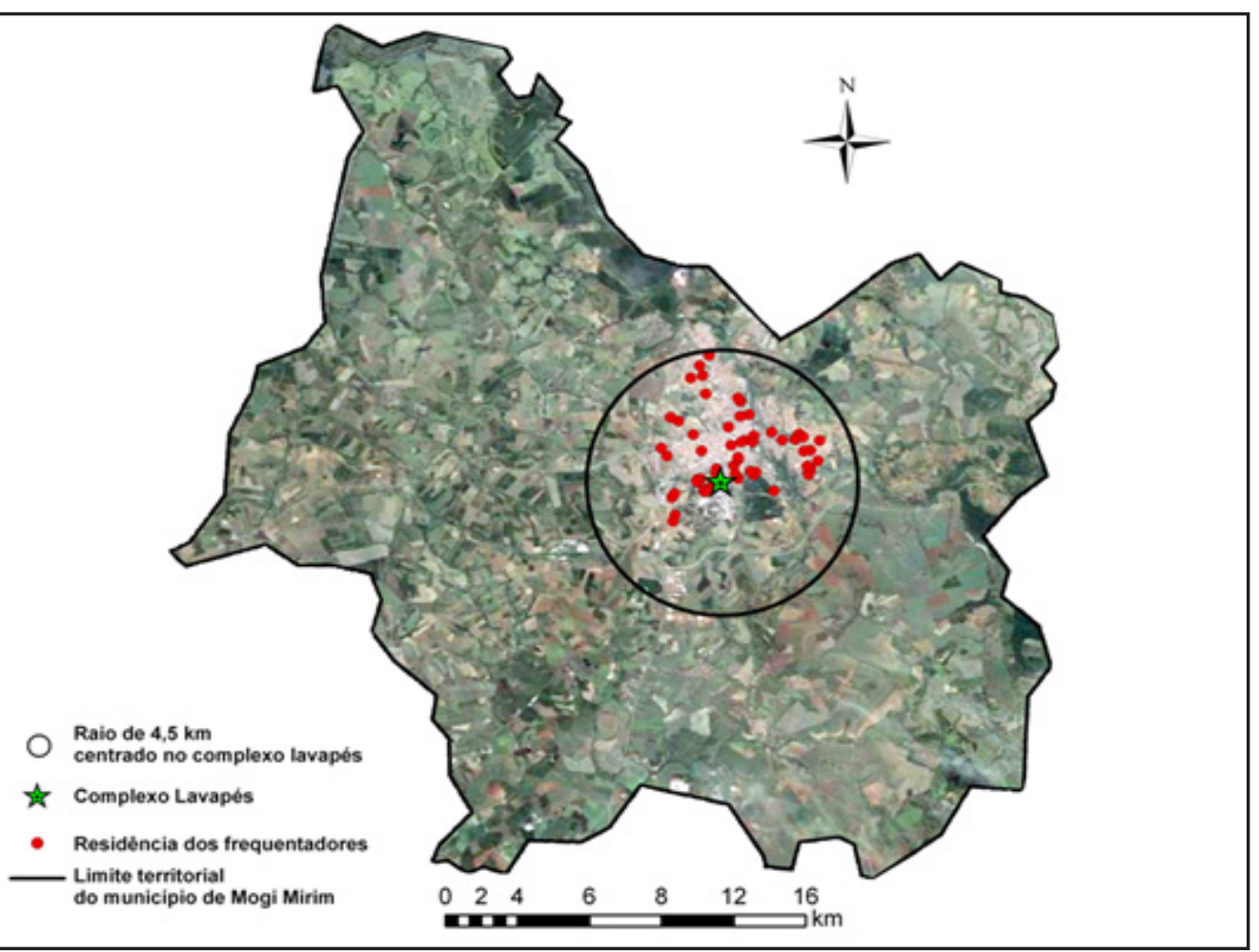

Figura 2: Localização espacial dos frequentadores da AV Complexo Lavapés no município de Mogi Mirim, em um raio de 4,5 km, baseada em pesquisa conduzida entre fevereiro e agosto de 2012.

Tabela 2 - Análise univariada apresentando as razões de chances, os intervalos de confiança (IC a 95\%) e o valor de p para as variáveis explanatórias com frequência de uso de área verde de no mínimo uma vez por semana, para os dados coletados entre fevereiro e agosto de 2012 na cidade de Mogi Mirim, SP.

\begin{tabular}{lccc}
\hline \multicolumn{1}{c}{ Variáveis } & RC & IC 95\% & p \\
\hline Ter cão & 1,00 & - & 0,01 \\
\hline Não & 1,92 & $1,16-3,16$ & \\
Sim & & & 0,01 \\
\hline Ter jardim em casa & 1,00 & & \\
\hline Não & 1,95 & $1,16-3,26$ & 0,02 \\
Sim & & & \\
\hline Meio de locomoção por ônibus & 1,00 & & \\
\hline Não & 0,52 & $0,30-0,90$ & 0,04 \\
Sim & & & \\
\hline Ter horta em casa & 1,00 & $1,05-5,29$ & \\
\hline Não & 2,36 & & \\
Sim & & & \\
\hline
\end{tabular}


...continuação - Tabela 2

\begin{tabular}{lcc}
\hline Usa medicamento para hipertensão & & \\
\hline Não & 1,00 & 0,06 \\
Sim & 0,57 & $0,31-1,04$ \\
\hline Escolaridade & & - \\
\hline Fundamental & 1,00 & $0,38-1,16$ \\
Médio & 0,67 & 0,10 \\
Superior & 0,48 & $0,24-0,97$
\end{tabular}

Meio de locomoção por automóvel

\begin{tabular}{|c|c|c|c|}
\hline Não & 1,00 & - & 0,10 \\
\hline Sim & 1,50 & $0,92-2,45$ & \\
\hline
\end{tabular}

\section{Gênero}

Masculino

1,00

0,19

Feminino

1,38

$0,85-2,25$

Auto avaliação da saúde

Ruim 1,00

$1,74 \quad 0,71-4,25$

Boa / muito boa

1,00

0,24

Ter área verde em casa

Não

1,35

$0,82-2,21$

Sim

\begin{tabular}{|c|c|c|c|}
\hline Não & 1,00 & - & 0,24 \\
\hline Sim & 1,66 & $0,71-3,88$ & \\
\hline \multicolumn{4}{|c|}{ Meio de locomoção por bicicleta } \\
\hline Não & 1,00 & - & 0,26 \\
\hline Sim & 1,53 & $0,73-3,22$ & \\
\hline \multicolumn{4}{|c|}{ Tempo de moradia (anos) } \\
\hline$<2$ & 1,00 & - & 0,39 \\
\hline$\geq 2$ & 1,00 & $0,99-1,00$ & \\
\hline \multicolumn{4}{|c|}{ Ter filho de até 10 anos } \\
\hline Não & 1,00 & - & 0,39 \\
\hline Sim & 0,78 & $0,45-1,37$ & \\
\hline
\end{tabular}

continua... 


\begin{tabular}{|c|c|c|c|}
\hline Não & 1,00 & - & 0,46 \\
\hline Sim & 1,36 & $0,60-3,06$ & \\
\hline \multicolumn{4}{|c|}{ Ter pomar em casa } \\
\hline Não & 1,00 & - & 0,46 \\
\hline Sim & 1,37 & $0,59-3,19$ & \\
\hline Idade (anos) & 1,00 & $0,99-1,02$ & \\
\hline \multicolumn{4}{|c|}{ Meio de locomoção a pé } \\
\hline Não & 1,00 & - & 0,60 \\
\hline Sim & 0,87 & $0,52-1,47$ & \\
\hline \multicolumn{4}{|c|}{ Meio de locomoção por motocicleta } \\
\hline Não & 1,00 & - & 0,61 \\
\hline Sim & 1,28 & $0,50-3,26$ & \\
\hline \multicolumn{4}{|c|}{ Índice de massa corporal (kg.m-2) } \\
\hline$\geq 25$ & 1,00 & - & 0,65 \\
\hline$<25$ & 0,89 & $0,54-1,46$ & \\
\hline \multicolumn{4}{|l|}{ Tabagismo } \\
\hline Não & 1,00 & - & 0,87 \\
\hline Sim & 1,05 & $0,57-1,95$ & \\
\hline
\end{tabular}

O resultado da análise multivariada pode ser observado na Tabela 3.

Tabela 3 - Análise multivariada apresentando as razões de chances, os intervalos de confiança (IC 95\%) e o valor de p para as variáveis explanatórias com frequência de uso de área verde de no mínimo uma vez por semana, para os dados coletados entre fevereiro e agosto de 2012 na cidade de Mogi Mirim, SP.

\begin{tabular}{|c|c|c|c|}
\hline Variáveis & $\mathbf{R C}$ & IC $95 \%$ & $\mathbf{P}^{*}$ \\
\hline \multicolumn{4}{|l|}{ Ter cão (TC) } \\
\hline Não & 1,00 & - & 0,016 \\
\hline Sim & 1,88 & $1,13-3,15$ & \\
\hline \multicolumn{4}{|l|}{ Ter jardim em casa (TJC) } \\
\hline Não & 1,00 & - & 0,025 \\
\hline Sim & 1,83 & $1,08-3,11$ & \\
\hline \multicolumn{4}{|c|}{ Meio de locomoção por ônibus (MLO) } \\
\hline Não & 1,00 & - & 0,018 \\
\hline Sim & 0,51 & $0,29-0,89$ & \\
\hline
\end{tabular}


Assim, o modelo multivariado pode ser expresso pela Equação 4:

$$
y=-0,055+0,6319(T C)+0,6058(T J C)-0,6736(M L O)
$$

Pela Equação 4 e a aplicação da Equação 3, sujeitos que têm cão, possuem jardim em casa e não usam o ônibus como meio de locomoção apresentam $77 \%$ de probabilidade em frequentar áreas verdes no mínimo uma vez por semana. No outro extremo, com a menor probabilidade de $33 \%$, estão aqueles sem cão, sem jardim e que usam ônibus.

A posse de um cão e a elevação de chance de frequentar uma área verde, como encontrado neste estudo, está em consonância com a literatura revisada. No estudo sobre uso de áreas verdes na Dinamarca, concluiu-se que ter um cão está associado à frequência de idas às áreas verdes ${ }^{6}$. A influência desta variável pode ser explicada pela necessidade do cão em se exercitar, na disponibilidade de espaço oferecido pela área verde e na companhia ou segurança que o animal proporciona.

A variável referente ao cultivo de um jardim na residência pode refletir uma preferência dos moradores em estarem próximos ou em contato com a natureza. Possivelmente por esta razão ela foi considerada significante no modelo final, uma vez que a chance de frequentar a área verde dos indivíduos que possuem jardim é de 1,83 vezes a dos que não adotam esta prática ou estilo de vida.

$\mathrm{Na}$ literatura revisada não foi encontrada nenhuma pesquisa que contemple esta variável associada ao uso da área verde pública. Existem estudos mostrando associação entre jardins comunitários e bem estar físico e psicológico. Os benefícios são atribuídos a diversos fatores, entre eles a atividade física praticada pela jardinagem, no entanto pode haver confusão com relação a influência do espaço verde na saúde e a seleção por indivíduos mais saudáveis em escolherem viver em áreas com espaços verdes $^{14}$.

Na Holanda, um estudo em grande escala epidemiológica (17 mil pessoas) mostra associação positiva entre disponibilidade e volume de área verde residencial (casa e bairro) e melhor condição de saúde ${ }^{15}$.

O uso de ônibus como meio de transporte se revelou como um fator de desestímulo à frequência em áreas verdes. O resultado apontou que a chance destes cidadãos é de 0,51 vezes comparando-se com aqueles que não fazem uso desta opção de transporte. Supõe-se que tais usuários, pelo uso de transporte coletivo, se sintam menos dispostos, pois a viagem corresponderia a uma extensão da jornada de trabalho diária. Além disso, esses indivíduos usualmente necessitam fazer caminhada para utilizar este meio de transporte.

Os resultados obtidos nesta análise corroboram os achados de outros estudos ${ }^{8,16}$, pelos quais se observa que indivíduos com maior mobilidade, ou que possuam condução própria, frequentam mais áreas verdes em Bristol, Inglaterra, ou o fato de não possuir automóvel funciona como uma barreira, desmotivando o acesso a áreas verdes.

O fato de usuários de ônibus frequentarem menos espaços verdes pode demonstrar a necessidade em melhorar o acesso às áreas verdes para população de menor poder econômico.

\section{CONCLUSÃO}

Este estudo buscou identificar os fatores sociodemográficos, de amostra de residentes em Mogi Mirim, associados à frequência de áreas verdes no município, no contexto amplo da promoção da saúde. Parte expressiva dos frequentadores faz uso desses espaços para prática de atividade física e convívio social.

Os resultados deste trabalho mostraram que diferentes fatores estão associados ao uso de áreas verdes. Possuir cão e ter jardim na 
residência são fatores motivadores, enquanto que utilizar ônibus, como principal meio de transporte, é considerado como desmotivador. Poderiam estas variáveis encontradas apontar alguma diferença entre classes sociais na frequência de uma área verde? Escolaridade, que seria uma variável que se aproximasse como indicador, não apresentou significância no estudo.

Usar o ônibus como principal meio de transporte, por exemplo, pode indicar, nas circunstâncias do padrão da sociedade brasileira, um indicador destas diferenças, em especial quando esta variável é significante como desestímulo.

Recomenda-se a condução de estudos posteriores que caracterizem as residências dos sujeitos participantes da pesquisa, sua inserção em classe social e as áreas verdes locais (quantidade e tipo de vegetação, estrutura oferecida, manutenção, distância e segurança) sejam conduzidos com o fim de oferecer subsídios para políticas públicas. Assim, uma perspectiva de planejamento urbano, considerando as especificidades da população local e o efeito das áreas verdes no bem-estar dos indivíduos poderia ser uma estratégia vigorosa de promoção em saúde.

Esta pesquisa explorou e apresentou uma metodologia de pesquisa para avaliação das características socioeconômicas da população e possíveis associações com uso de AV. Desse modo, consiste em um recurso expressivo, pouco abordado na literatura nacional, que deve ser reproduzido com o fim de estimular esta prática essencial de promoção à saúde.

\section{REFERÊNCIAS}

1. IBGE - Instituto Brasileiro de Geografia e Estatística. Censo Demográfico 2010. Disponível em: < http://www.ibge.gov.br/cidadesat/ topwindow.htm?1>. Acesso em: 23 agosto 2011.

2. Loboda CR, De Angelis BLD. Áreas verdes públicas urbanas: conceito, usos e funções. Ambiência - Rev Centro Ciências Agrárias Amb.2005; 1:125-139.

3. Caporusso D, Matias LF. Áreas verdes urbanas: avaliação e proposta conceitual.In: Seminário de Pós-Graduação Em Geografia Da Unesp, 8., 2008, Rio Claro. Anais do I Simpósio de Pós-graduação em Geografia do Estado de São Paulo, Rio Claro, 2008, p. 71-87

4. Vieira, PBH. Uma visão geográfica das áreas verdes de Florianópolis, SC: estudo de caso do Parque Ecológico do Córrego Grande (PECG). Trabalho (Conclusão de curso), Universidade Federal de Santa Catarina, Florianópolis - SC, 2004.

5. Lima AMLP, Cavalheiro F, Nucci JC, Souza MAB, Fialho NO, Del Picchia PCD. Problemas de utilização na conceituação de termos como espaços livres, áreas verdes e correlatas. In: Ii Congresso Brasileiro de Arborização Urbana, 2., São Luís. Anais... São Luís: Imprensa EMATER/MA, Setembro de 1994.

6. Schipperijn J, Stigsdotter UK, Randrup TB, Troelsen J. Influences on the use of urban green space - A case study in Odense, Denmark. Urbn For Urban Green. 2010; 9:25-32.

7. Grahn P, Stigsdotter U. Landscape planning and stress. Urb For Urban Green. 2003; 2:1-18.

8. Kessel A, Green J, Pinder R, Wilkinson P, Grundy C, Lachowycz K. Multidisciplinary research in public health: A case study of research on access to green space. Pub Health. 2009; 123: 32-40.

9. Richardson EA, Mitchell R. Gender differences in relationships between urban green space and health in the United Kingdom. Soc Sci Med. 2010; 71: 568-575.

10. Marino LMR. Caracterização e zoneamento ambiental do zoológico municipal de Mogi Mirim-SP. 2008. Tese (Doutorado) Universidade Federal de São Carlos, UFSCar, 2008.

11. SISVAN. Sistema de Vigilância Alimentar e Nutricional, DATASUS, Ministério da Saúde, 2013. Disponível em: http://tabnet.datasus. gov.br/cgi-win/SISVAN/CNV/notas_sisvan.html. Acesso em: 07 março 2013.

12. Gil AC. Metodologia da pesquisa. São Paulo: Atlas, 1995.

13.Hosmer DW, Lemeshow S. Applied logistic regression. Wiley Interscience, New York, 1989. p.31-186.

14. Groenewegen PP, Van Den Berg A, Vries S, Verheij RA. Vitamin G: effects of green space on health, well-being, and social safety. BMC Pub Health. 2006; 6: 149-158.

15. De Vries S, Verheij RA, Groenewegen PP, Spreeuwenberg P. Natural environments - healthy environments? An exploratory analysis of the relationship between greenspace and health. Environ Plan. 2003; 35: 1717-31.

16. Jones A, Hillsdon M, Coombes E. Greenspace access, use, and physical activity: Understanding the effects of area deprivation. Prev Med. 2009; 49: 500-505. 